Proceedings of the 31th Annual Meeting of the Brazilian Embryo Technology Society (SBTE); Cabo de Santo Agostinho, PE, Brazil, August 17th to 19th, 2017.

\title{
Negative energy balance and metabolic stress in relation to oocyte and embryo quality: an update on possible pathways reducing fertility in dairy cows
}

\author{
Jo L.M.R. Leroy ${ }^{1}$, Jessie de Bie, Lies Jordaens, Karolien Desmet, Anouk Smits, Waleed F.A. Marei, Peter \\ E.J. Bols, Veerle Van Hoeck
}

Gamete Research Centre, University of Antwerp, Wilrijk, Belgium.

\begin{abstract}
A negative energy balance in metabolically compromised high producing dairy cows has been shown to influence oocyte and embryo quality. However, the possible involved pathways needed more attention to better understand specific deleterious effects. Oocyte maturation is the first process to be scrutinized. Because many possible metabolic factors might directly impact oocyte quality, systematic in vitro approaches were used to investigate the effects of oocyte maturation under elevated NEFA concentrations. Blastocysts originating from NEFA-exposed oocytes showed a lower cell number, an increased apoptotic cell index, signs of glucose intolerance, sensitive to oxidative stress and mitochondrial dysfunction. Defining these embryos' transcriptome and epigenome signatures revealed changes in DNA methylation patterns. Long-term exposure of developing murine follicles to elevated NEFA concentrations showed to impair oocyte developmental competence even more. While little is known on how the oviductal microenvironment can change as a consequence of a negative energy balance, a validated in vitro bovine oviduct model offered some valuable insights on how NEFAs disturb oviductal cell physiology. NEFA exposure reduces cell proliferation, cell migration, sperm binding capacity and monolayer integrity. In addition, oviductal cells seem to play an active role in regulating luminal NEFA-concentrations through increased permeability, intracellular lipid accumulation and fatty acid metabolism. This might favour early embryo development. The establishment of a successful pregnancy largely depends on the ability of the embryo to interact with a properly prepared endometrium. Because suboptimal physiological conditions influence oocyte maturation and embryo development to the extent that epigenetic consequences are unavoidable, the question arises if these changes hamper embryo implantation and subsequent development. Gene expression studies on epithelial endometrial cells brought in contact with in vitro embryos cultured for 4 days under suboptimal conditions reveal that the embryo-endometrial signaling is affected. Transfer of bovine embryos derived from compromised oocytes showed disturbed embryo development following recovery at day 14 with a negative impact on IFNt secretion and therefore suggesting carry-over effects from suboptimal culture conditions. The current paper will document the most important recent findings and comment on extrapolation possibilities from in vitro
\end{abstract}

studies to field conditions in daily dairy practice. In addition, the possibility of remediating approaches will be discussed to see how this knowledge might generate insights on possible alleviating strategies.

Keywords: embryo, follicle, maternal metabolic disorders, oocyte, peri-conception, subfertility.

\section{Introduction}

Mammals should reproduce to be able to lactate. Scientists, advising nutritionists and veterinarians are now aware of the fact that dairy cows should reproduce within a limited time span after calving to obtain the best economical results. Dairy cows are, from an economical point of view, the best producers during and just after peak lactation. Only fertile cows will have several of these efficient periods in their productive live span. It is not always easy to convince our dairy farmers of the central role "dairy cow fertility" plays in generating a substantial income, in sustainability and in the environmental impact of their dairy business. The fertility "story" is difficult to tell because it is not easy to measure reproductive performance of an individual cow or a dairy herd (in contrast to for example milk production performances) in a sound way (for review: Leblanc et al., 2010). Many heavily confounded fertility indices have been used (and are still routinely reproduced) to advice farmers, leading to no or wrong management decisions. Furthermore, this resulted in difficulties benchmarking farms or in judging the real gain of improved management strategies on fertility. When we discuss fertility with farmers it is not always clear whether we focus on the inherent reproductive ability of the cow's body (physiology) and/or on the reproductive performance of that specific cow kept under specific management conditions. It is very important to know the difference, as it will determine whether a cow and/or a cow's management should be "treated". Optimal reproduction and the generation of healthy offspring is the result of a very long chain of finely tuned physiological processes. Everything starts with the formation of the primordial germ cells in the embryo early during pregnancy and should lead to the successful ovulation and fertilization of a top quality oocyte, finally resulting in an ongoing pregnancy culminating in successful birth of a healthy offspring. Numerous factors affect this intricate long play of reproductive physiology and will determine the cow's reproductive efficiency. Apart from the genetic trait effects, the nurture effects on reproductive 
capacities start in utero, and show to be also important during the calf and heifer rearing period (GonzalesRecio et al., 2012; Lohakare et al. 2012; Sinclair et al., 2016). Many studies focused already on the effect of the conditions during the dry and the transition period on reproduction. Dry matter intake, feed composition, social stress, housing conditions have all been associated with reproductive outcome (Wathes et al., 2012. Furthermore, awareness grows on the direct link between metabolic diseases in the dairy cow and the concomitant subfertility issue (Leroy et al., 2008). Numerous studies come up with conflicting results, but the most important generally agreed conclusion is that the metabolic health of the cow directly impacts on fertility. This is because the somatotropic axis affects the fertility axis at several levels, disturbing the endocrine interplay of female reproduction and thereby hampering timely ovulation. Furthermore, more and more data appear on how maternal metabolism directly affects oocyte and embryo quality due to changes in their micro-environment. For some years now, our laboratory focused on the pivotal role of the oocyte and the pre-implantation embryo in the link between maternal metabolic health and fertility. This review aims to provide an overview on the most recent advances and on the new insights this research has yielded by using the research model as presented in Fig. 1.
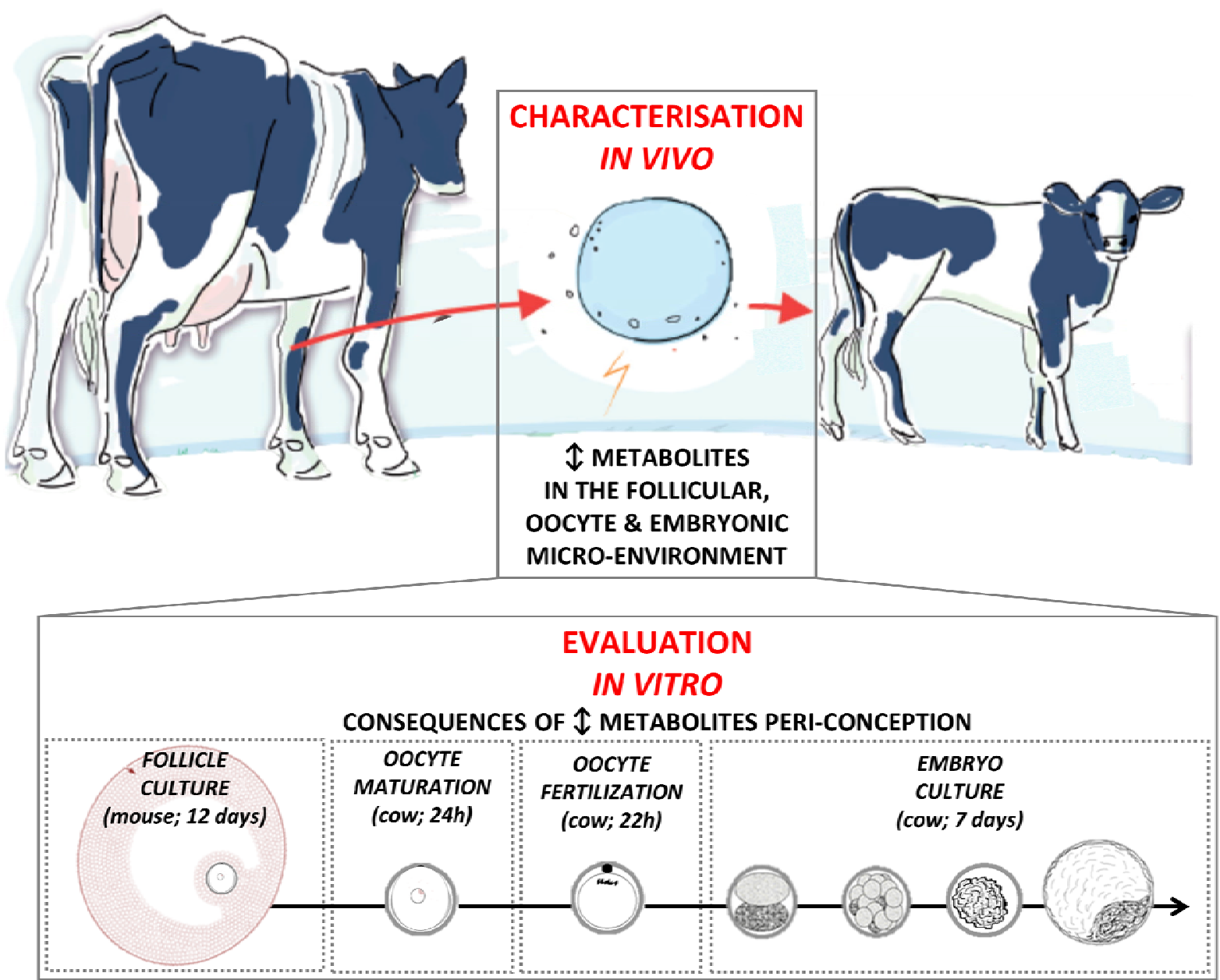

Figure 1. Overview of the general research model from which all data, described in this review, originate.

\section{How sensitive is the oocyte during the process of maturation?}

The oocyte and embryo are of questionable quality in females suffering lipolytic disorders. Sartori et al. (2002) and Leroy et al. (2005) showed that the proportion of viable embryos is drastically reduced in lactating cows compared to non-lactating cows or heifers. More than $40 \%$ of the dairy cow conceptuses are lost within two weeks post-insemination (Leroy et al., 2008). Based on the known metabolic changes in the blood during periods of metabolic stress, several 'candidate' metabolic factors have been proposed to directly impact on fertility. Meticulously designed lab animal studies are, however, needed to distinguish whether the observed effect is exerted at the level the oocyte, zygote, embryo or sperm cell, or rather a combination of the latter. Hereto, when using in vitro oocyte maturation models, which allow to mimic metabolic disorders at the level of the oocyte, metabolite concentrations should be based on intrafollicular rather than on serum concentrations (Leroy et al., 2004). One of the best-studied examples of metabolic changes in follicular fluid is the negative 
energy balance (NEB) condition in the high-yielding dairy cow (Leroy et al., 2008). These insights provide the basis for translation of metabolic disorders from the in vivo follicular environment towards our in vitro wells. Based on the known intra-follicular metabolite changes, during NEB periods of metabolic stress, several "candidate" factors have been extensively tested using our bovine and murine in vitro oocyte maturation models.

For example, effects of high free fatty acid (NEFA) concentrations during the final phase of oocyte maturation have been tested. Using a bovine in vitro model, we examined the effect of oocyte maturation in the presence of patho-physiologically relevant high concentrations of the three most important NEFAs (palmitic, stearic and oleic acid). We learned that final oocyte maturation under elevated NEFA concentrations $(425 \mu \mathrm{m})$ results in blastocysts with a significantly lower cell number and increased apoptotic cell index (Van Hoeck et al., 2011). Surprisingly, such embryos displayed glucose intolerant characteristics, were triggered by oxidative stress regulating mechanisms and showed signs of mitochondrial dysfunction. Gene expression and functional data suggest that the resulting embryos have altered metabolic strategies, which might explain the aberrant energy metabolism and suggests a mechanism of metabolic dysregulation appearing in the pre-implantation embryo as a consequence of elevated NEFA concentrations peri-conception (Van Hoeck et al., 2013). All details of the latter studies are compiled in Table 1.

Furthermore, it was questioned whether glucose availability in the oocyte's micro-environment could intensify the observed effect of the high NEFA exposure. A lipolytic state in maternal metabolic disorders may also be accompanied by hypoglycemia during a NEB status in dairy cows (Leroy et al., 2008). Therefore, the effects of high and low glucose concentrations (9.9 and $2.8 \mathrm{mM}$, respectively) in the presence of elevated NEFAs $(420 \mu \mathrm{m})$ during oocyte maturation were tested on oocyte and embryo quality, metabolism and developmental competence. Interestingly, in case of limited glucose availability, the effect of the high NEFA exposure on cumulus cell expansion and oocyte development was more prominent then when NEFAs were added in combination with high glucose concentrations. However, both conditions resulted in surviving embryos of reduced quality regarding to blastomere apoptosis and cell allocation (De Bie et al., 2017).

Step by step, based on the above described data, awareness grows that altered metabolic conditions during oocyte maturation alters transcriptome and epigenome signatures of the resulting blastocysts. Indeed, oocyte maturation under elevated NEFA concentrations significantly up-regulated the gene expression of DNMT3A both in the matured oocyte and in the day 7.5 blastocysts after routine fertilization and culture (Van Hoeck et al., 2013). The DNMT3A gene encodes a "de novo DNA methyltransferase", whose regulation is essential for the proper establishment of epigenetic marks (Van Hoeck et al., 2013). Further focus on elevated NEFA concentrations during oocyte in vitro maturation reveal influenced DNA methylation patterns in the resultant blastocysts, although to a relatively limited extent (Desmet et al., 2016b). Key cellular pathways affected by NEFA exposure were similar after the integration of gene expression and methylation patterns, with particular reference to lipid and carbohydrate metabolism, cell death, immune response and metabolic disorders (Desmet et al., 2016b). Thereby, metabolic perturbations, induced in the oocyte, may not simply result in suboptimal conception rates, but can also result in persisting affects during fetal development or become visible after birth as stated by Vickers (2014).

Finally, it is important to keep in mind that before entering the oviduct, the oocyte has spent several months to years in the ovary. Most of the in vitro studies performed so far, describe effects of suboptimal nutrient environments during the final oocyte maturation period under suboptimal metabolite conditions for merely 24 h. The physiological relevance of the highly defined $24 \mathrm{~h}$ in vitro maturation exposure model is therefore debatable. Therefore, a murine model was used to study the effect of long-term elevated NEFA concentrations on the follicle as a whole. This model includes the individual culture of early secondary preantral follicles up until day 13, when the in vitro follicles reach the antral stage, followed by oocyte isolation, in vitro fertilization and embryo culture. The follicle culture has been validated as a functional follicular unit, much resembling the in vivo situation (Cortvrindt and Smitz, 1998). In this model, in vitro cultured murine follicles exposed to elevated NEFA concentrations resulted in an altered follicular physiology, only moderately affecting follicular growth and antrum formation (Valckx et al., 2013). Importantly, when these follicles are exposed to elevated NEFA concentrations throughout follicle growth or only during the final maturation phase, it was shown that long term exposure (13 days) more severely impairs oocyte developmental competence, compared to short term NEFA exposure (only during the final phase of oocyte maturation; Valckx et al., 2015). These data strengthen our impression that not only final oocyte maturation, but also the preceding period of follicular and oocyte growth is pivotal for oocyte developmental competence. Indeed, 25 years ago, Britt (1992) already hypothesized that follicles developing and growing during the period of NEB early post-partum could be affected by the unfavorable metabolic changes and therefore may contain a developmentally incompetent oocyte at the time of ovulation after the voluntary waiting period. Only recently, Carvalho et al. (2014) studied 70 cows and showed that cows significantly losing body weight during the first three weeks of lactation displayed a dramatically lower number of viable and transferable good quality embryos after a superovulation treatment around 100 days post-partum. These results are obviously consistent with the hypothesis proposed by Britt and, up till the report of Valckx et al. (2015), a potential clarifying mechanism was lacking. 


\section{What happens with the developing zygote during the first days after fertilization?}

In cattle, the early embryo resides in the oviduct for 4 days during which the embryo is highly sensitive to environmental changes (Latham and Schultz, 2001). Little is known about "whether and how" maternal metabolic disorders can modulate the embryonic micro-environment. Therefore, Jordaens et al. (2015) used a two-compartment polarized cell culture technique with hanging inserts (Miessen et al., 2011) as attempt to evaluate whether metabolic changes in the blood of mothers affect oviductal cell features and thereby potentially jeopardize the micro-environment of the early embryo. Results show that elevated NEFAs can affect in vitro bovine oviduct epithelial cell (BOEC) physiology by reducing cell proliferation, cell migration capacity, BOEC sperm binding capacity and monolayer integrity, in a cell polarity dependent manner (Jordaens et al., 2015). When studying these effects into closer detail (Jordaens et al., 2017), it was noticed that, when NEFAs were supplemented for $24 \mathrm{~h}$ in the basal (blood) compartment, their concentrations decreased in the basal compartment with a concomitant increase in the opposing apical chamber, indicating fatty acid transfer. Fascinatingly, when the $24 \mathrm{~h}$ NEFA-administration was performed at the apical side of the oviductal lumen, which will form the micro-environment of the embryo, no fatty acid transfer from apical to basal compartment could be observed, but intracellular lipid accumulation increased massively. Thus, apically administered NEFAs also induced anti-apoptotic and anti-oxidative pathways in the oviductal cells. These data suggest that BOECs may clear the micro-environment of the preimplantation embryo from luminal NEFAs through increased monolayer permeability, intracellular lipid accumulation and fatty acid metabolism. Thereby, in order to 'safeguard the embryo, the oviduct is suggested to perform a gatekeeper function to modulate its microenvironment in favor of the early embryo by alleviating potential lipotoxic effects (Jordaens et al., 2017).

However, it remains debatable whether this protective/clearing effect is sufficient, to actually protect in vivo embryos passing the oviduct from females suffering metabolic disorders. Recently, our research group investigated whether and to which extent plasma NEFAs are reflected in oviduct fluid of healthy cattle using an ex vivo flushing method. Interestingly, oviductal fluid and plasma NEFA concentrations did not differ significantly and tended to be positively correlated. Thereby, we can conclude that a tight regulation of the embryonic milieu might not be assured in females suffering metabolic disorders (Jordaens et al., 2017). Of course, ongoing research is further studying how the oviductal environment is affected in maternal metabolic stressed conditions. This can become an important threat in females suffering metabolic disorders. Indeed, several in vitro embryo culture studies, in which embryos were exposed to distinct suboptimal metabolite conditions, show that a pre-implantation embryo is very sensitive to any perturbation in its micro-environment.
For example, our data show that bovine embryos exposed to elevated NEFA concentrations during the in vitro culture period have a lower developmental potential (Van Hoeck et al., 2012). Based on this information, a recent study furthermore examined underlying pathways in bovine embryos based on a genome-wide microarray analysis (Desmet et al., 2016b). Details of the latter studies are compiled in Table 1. Overall, expression of different genes was affected, with particular focus on oxidative metabolism, apoptosis, endoplasmic reticulum stress and lipid metabolism. Furthermore, vast epigenetic erasure and reprogramming events take place during early embryogenesis (Dean et al., 2001; Smith et al., 2012), making the embryo also susceptible for epigenetic alterations. More in-depth research indeed confirms this by showing changes in DNA methylation after elevated NEFA exposure during embryo culture. The latter methylation data cover similar pathways as described for the, earlier documented, transcriptomic alterations (Desmet et al., 2016b). This implies that suboptimal metabolite conditions can directly influence epigenetic reprogramming in the embryo and affect its genetic signature.

Furthermore, combined in vivo and in vitro studies were used to investigate the effect of dietary induced hyperlipidemia during embryo culture. Overall, addition of hyperlipidemic serum rich in saturated NEFAs during bovine in vitro embryo culture significantly reduced embryo development and quality (Leroy et al., 2010). Importantly, the extent of the observed effects seems to depend on the fatty acid types involved. Indeed, changing the predominant fatty acid to an unsaturated type in hyperlipidemic serum differentially influenced embryo development (Marei et al., 2016). More specifically, both saturated fat rich and unsaturated fat rich diets induced hyperlipidemia and increased total serum NEFAs by $>33 \%$ in non-lactating cows, however, saturated serum was rich in palmitic acid while unsaturated serum was rich in alfa-linoleic acid (ALA). Addition of saturated hyperlipidemic serum during the in vitro embryo culture decreased blastocyst rates compared to control serum and surviving blastocysts had increased apoptosis. In contrast, unsaturated serum resulted in normal embryo development and quality with up-regulated DNMT3A indicating a regulatory role in DNA methylation compared to control serum (Marei et al., 2016).

Finally, it is important to consider that the oviduct not only provides the early embryonic environment, but also regulates the selection of spermatozoa, sperm storage, sperm motility and guidance of spermatozoa towards the egg (Holt and Fazeli, 2010). So far, we tested effects of direct NEFA exposure on follicle cells, oocytes, oviductal cells and embryos. However, information on effects of suboptimal metabolite conditions on the male gamete passing the female reproductive tract remained lacking. Therefore, we investigated to which extent sperm cells are influenced by altered metabolic conditions, such as elevated NEFAs. No obvious effect on the bovine fertilization process itself was noticed since cleavage 
rates were not significantly affected after in vitro fertilization under high NEFA conditions. However, further embryonic development was hampered when fertilization occurred in the presence of high NEFAs. Interestingly, as shown in Table 1, elevated NEFAs had no influence on the fertilizing capacity of pre-exposed sperm, suggesting that NEFA-induced reduction in developmental competence is through alterations in oocyte quality but not through affecting sperm quality (Desmet et al., 2016a). More research is ongoing to investigate underlying mechanisms.

\section{The fate of the embryo upon arrival in the uterus.}

Years of intense research in our laboratory taught us that maternal metabolic changes can affect follicle health, oocyte development and even subsequent embryo quality and metabolism. This can help to explain why, in dairy cows, a great proportion of the embryos die before day 7 following insemination (Spencer, 2013). However, we also showed how bovine embryos may survive acute NEFA exposure probably due to an adaption in their metabolism (Van Hoeck et al., 2013, 2015). The latter does not inevitably guarantee that the embryo will implant successfully and lead to a full-term pregnancy. In cattle, embryonic loss peaks between days 7 and 16 post-mating during the period in which the uterus must develop receptivity in response to ovarian P4 to support conceptus growth and implantation (Spencer, 2013). Ayalon (1978) reported that critical period for embryonic death appears to be soon after the embryo enters the uterus. Indeed, upon arrival in the uterus a critical maternal-embryonic interplay needs to occur in order to fulfill: shedding of the zona pellucida, orientation, apposition, preattachment and adhesion of the blastocyst to the endometrium (Spencer et al., 2004). For the latter events, the differentiation fingerprint of both blastocyst and endometrial tissue will be of crucial importance. Suboptimal differentiation of the TE cell line, that becomes aligned against the endometrial epithelium, will compromise the success of the very first maternalembryonic interactions (Armant, 2005). The embryo will then not be able to surpass the critical preimplantation period in the uterus. Based on the latter insights, the question arises whether suboptimal metabolite conditions during oocyte and embryo culture can alter differentiation patterns in surviving embryos entering the uterus. Translation of recent crossdisciplinary insights might pave the way to understand how suboptimal maternal metabolite conditions could impact not only on the embryo till day $7-8$, but also on embryo survival beyond this timing and first embryonic-maternal interactions. Recent cancer cell studies revealed that regulation of cell differentiation occurs via nutrient-sensing mechanisms (Folmes et al., 2012). Therefore, the earliest pre-implantation phases of in vitro embryo development were studied as 'window' for nutrient sensitive manipulations. Embryos were cultured during the first 4 days after fertilization under distinct nutrient conditions (control, high glucose, low amino acids) and were transferred, at morula stage, on a monolayer of epithelial endometrial cells (BEEC) till day 8 p.i.. Gene expression data show that under suboptimal metabolite conditions during the first 4 days of embryo culture nutrient sensing programs in embryos are prompted through mTOR mediated pathways. This might be the mechanism through which suboptimal metabolic environments during the early embryonic reprogramming impact on resultant blastocyst cell proliferation and differentiation programs (Van Hoeck et al., 2016). Fascinatingly, when BEEC were placed in contact with the three distinct groups of embryos, BEEC transcriptome profiles were differently regulated. Regulation of the latter genes might be critical for creating a receptive state towards the embryo. Even more, in BEEC exposed to embryos cultured under suboptimal conditions, the expression of two IFNtresponsive genes was down-regulated. Finally, a decreased integrin gene expression in blastocysts from nutrient-sensed embryos, and the subsequent downregulated expression of cell adhesion factors in the allied BEEC, suggests that ligand-ligand interaction can be important for the first maternal-embryonic interactions. This further supports the importance of close contact between mother and embryo yet at this early timing, as recently stated by Sponchiado et al. (2017).

As stated above, there is a clear need for more studies focusing on post day 7 embryo development. In order to expand on this, we transferred morphologically equal day 7.5 blastocysts derived from NEFA-exposed or control oocytes to recipient cows and recovered the 14 day old embryos to investigate post-hatching development. Considering that the second week of development coincides, as explained above, with processes such as cell lineage specialization, embryo elongation and embryonic-maternal signaling (Berg et al., 2010), this post day 7 investigation is crucial. Preliminary data indicate that day 14 embryos derived from high NEFA-exposed oocytes were less developed after recovery compared to their control counterparts. Moreover, these embryos were metabolically compromised and had reduced IFNt secretion, a major signal of pregnancy recognition. This suggests that an insult during oocyte maturation may have long-lasting effects until the peri-implantation period, possibly affecting further development. Ongoing research focuses on the genome wide transcriptomic profiles using RNA sequencing techniques to discover potentially affected pathways.

\section{What did we learn so far that can be useful to improve fertility?}

Whether our in vitro models are suitable to withdraw/extrapolate conclusions towards the in vivo problem of the increased incidence in embryonic loss remains a matter for debate. By using such in vitro models, the awareness increased about whether and how suboptimal metabolite concentrations, such as elevated serum NEFA concentrations, are a potential threat around the period of conception (for an overview of peri-conception NEFA-toxicity; Table 1). 
Leroy et al. Metabolic health and bovine oocyte quality.

Table 1. The effects of elevated NEFAs during bovine in vitro oocyte maturation, fertilization or embryo culture.

HIGH NEFA EXPOSURE DURING FINAL BOVINE IN VITRO OOCYTE MATURATION (24 H)



EVALUATED STAGE 


\section{HIGH NEFA EXPOSURE DURING FINAL BOVINE IN VITRO OOCYTE FERTILIZATION (22 H)}

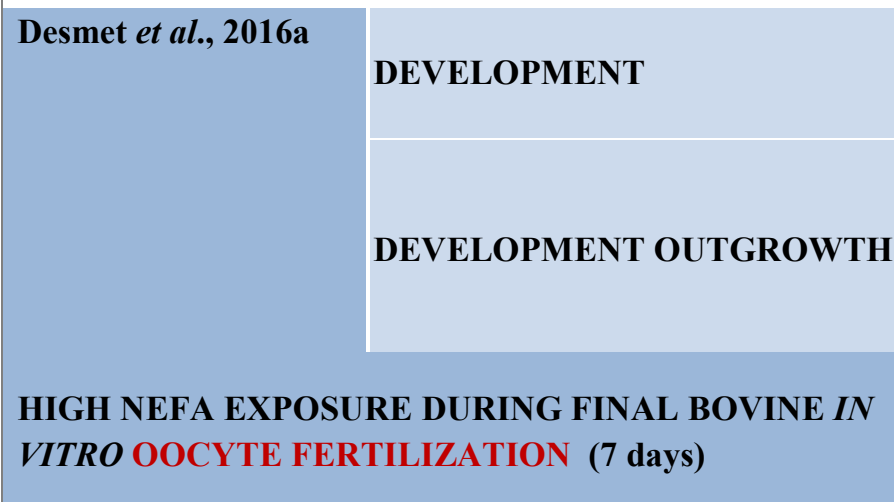

Desmet et al., 2016b

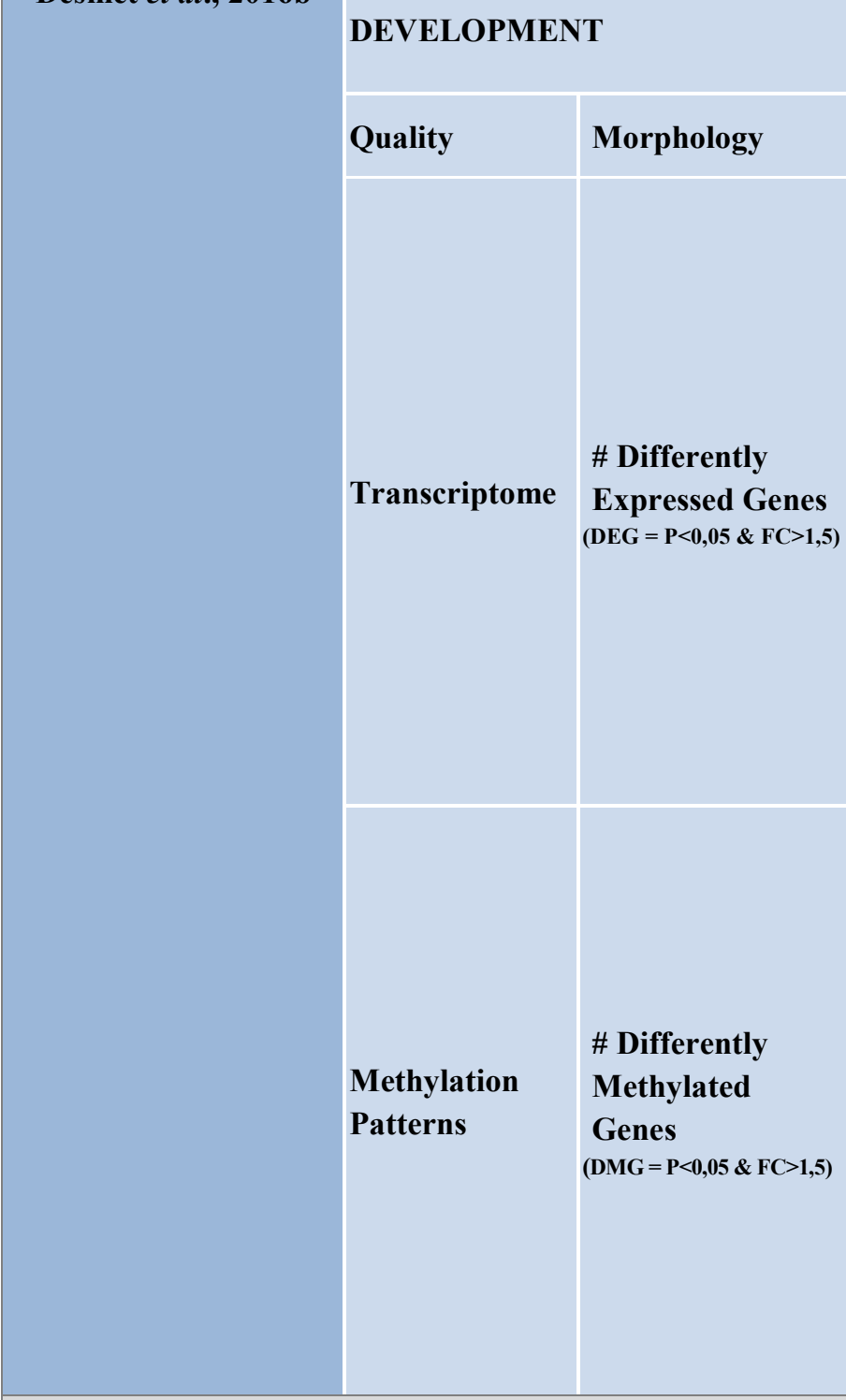

\begin{tabular}{|c|c|c|c|}
\hline \multicolumn{4}{|c|}{ EVALUATED STAGE } \\
\hline Oocyte & $\begin{array}{l}\text { 2-Cell } \\
\text { Zygote }\end{array}$ & Morula & Blastocyst \\
\hline & $\begin{array}{l}\searrow \text { cleavage } \\
\uparrow 2 \text {-cell block }\end{array}$ & & $\downarrow$ \\
\hline & & & $\begin{array}{c}\searrow \\
\text { Outgrowth } \\
\text { post day } 9\end{array}$ \\
\hline & EVALUAT & ED STAC & \\
\hline
\end{tabular}

\begin{tabular}{|c|c|c|c|}
\hline \multirow{2}{*}{ Oocyte } & $\begin{array}{c}\text { 2-Cell } \\
\text { Zygote }\end{array}$ & Morula & Blastocyst \\
\hline
\end{tabular}

$\downarrow$ cleavage

$206 \uparrow 105 \downarrow$

Major

pathways

affected

-cell

morphology

-cell-to-cell

signaling

-hematological

system

-lipid

metabolism

-small

molecule

biochemistry

1576 DMG

$697 \uparrow 879 \downarrow$

Major

pathways

affected:

-cell death and

survival

-lipid

metabolism

-carbohydrate

metabolism

-molecular

transport

-embryonic

development

$\downarrow$ significantly reduced compared to control $/ \searrow$ trend for reduction compared to control $\approx$ no difference compared to control / $\lambda$ trend for increase compared to control $/ \uparrow$ significant increase compared to control

However, while most in vitro studies, designed to carefully mimic disturbed metabolic conditions in the well, show drastic effects on oocyte quality, in vivo dairy cow studies are less univocal (Matoba et al., 2012). Differences in study design, environmental conditions, evaluated end-point parameter, genetic 
background and diet may explain this lack of uniformity.

Furthermore, when observing all data generated in our laboratory, one important question arises; is the damage observed during early embryonic culture a point of no return that will be imprinted in the embryo and will become visible later in life? Or are there compensating mechanisms that come into play during further embryo and fetal development that will erase all errors? Interestingly, the condition in which oocyte growth and maturation takes place is critical for its subsequent developmental capacity (Van Hoeck et al., 2011). However the micro-environment in which the embryo develops after fertilization is also important (Lazzari et al., 2010; Sermondade et al., 2012).

Therefore, we tested whether metabolicallycompromised bovine oocytes matured under elevated pathophysiological NEFA concentrations (and altered glucose availability), could be rescued by adding rescuing compounds to the subsequent embryo culture medium. Remarkably, detrimental effects of high NEFA concentrations during oocyte maturation on embryo development could be alleviated, at least in part, by supplementation of additives, such as serum or insulintransferrin-selenium, during culture. However, blastocysts were still inferior in quality, as evidenced by increased apoptosis, abnormal cell segregation patterns and altered metabolic behavior (Smits et al., 2016; De Bie et al., 2017).

Furthermore, also fatty acid saturation comes into play when considering potential interventions for recovery of metabolically compromised matured oocytes. Each type of NEFA and each ratio of saturated and unsaturated fatty acids seems to have its specific effects on the early stages in life, but also seems to be able, at least to some extent, to counteract effects of other NEFA types. However, to which extent such counteracting mechanisms are sufficient in order to sustain further development of the gamete (and subsequent embryo physiology) remains an intriguing issue. In this context, omega- 3 fatty acids may have a promising role in protecting maturing oocytes from lipotoxic insults and we showed that it is possible to enhance oocyte quality by reducing cellular stress levels by e.g. ALA supplementation during oocyte maturation (Marei et al., 2017). Therefore, dietary supplementation with ALA in cows might be a promising strategy to resolve subfertility problems that are associated with reduced oocyte quality due to metabolic disorders associated with upregulated lipolysis during periods of negative energy balance.

\section{Conclusions. Significance for the dairy manager}

Focusing on dairy cow fertility, pathological suboptimal metabolite conditions are proposed as a risk factor for disappointing reproductive performance. Years of expertise in animal in vitro embryo culture models contribute to the awareness that a normal maternal 'metabolic health' status is essential to safeguard successful ovulation, conception and further embryo development. In this context, high NEFA concentrations in the blood are known to alter the follicular and most probably also the oviductal microenvironment. The latter alterations in NEFA concentrations have been associated with a disappointing fertility outcome through disabled ovarian cell function, reduced oocyte's developmental competence and even embryonic transcriptome, metabolome and phenotypic fingerprints. Strategies should thus be based on alleviating suboptimal metabolite concentrations in the blood, and thereby aim to create optimal nutrient environments for the oocyte and embryo. Multiple "symptomatic" efforts have been done by designing dietary strategies to improve metabolically stressed condition of dairy cows. However, it is hard to come up with practical guidelines. It is generally accepted that the nutritional requirements for early resumption of ovarian activity and follicular growth are different from the nutritional conditions optimal for conception and early embryo growth. In this context, one should reflect on the believe that attempts to alleviate metabolic disorders, such as NEB status in the cow, are not always compatible with attempts to create optimal nutrient environments for the oocyte during final follicular growth and embryo during the first steps of development. Furthermore, besides focusing on the oocyte and early embryonic environment, one should consider the uterine environment which needs to prompt first maternal-embryonic interactions. Based on our first in vitro co-culture data, in which we detected different responses in BEEC allied with distinct types of 'nutrient-sensed' embryos, the emerging hypothesis raised that the endometrium is an active participant of successful pre-implantation embryo development. This reflection provides a new approach towards strategies that are highly needed to improve efficiency of fertility treatments.

\section{References}

Armant DR. 2005. Blastocysts don't go it alone. Extrinsic signals fine-tune the intrinsic developmental program of trophoblast cells. Dev Biol, 280:260-280.

Ayalon N. 1978. A review of embryonic mortality in cattle. J Reprod Fertil, 54:483-493.

Berg DK, van Leeuwen J, Beaumont S, Berg M, Pfeffer PL. 2010. Embryo loss in cattle between days 7 and 16 of pregnancy. Theriogenology, 73:250-260.

Britt JH. 1992. Impacts of early postpartum metabolism on follicular development and fertility. In: Proceedings of the 24th Annual Convention of the American Association of Bovine Practitioners, 1991, Orlando, FL. Ashland, OH: AABP. pp. 39-43.

Carvalho PD, Souza AH, Amundson MC, Hackbart KS, Fuenzalida MJ, Herlihy MM, Ayres H, Dresch AR, Vieira LM, Guenther JN, Grummer RR, Fricke PM, Shaver RD, Wilbank MC. 2014. Relationships between fertility and postpartum changes in body condition and body weight in lactating dairy cows. $J$ Dairy Sci, 97:3666-3683.

Cortvrindt R, Smitz J. 1998. Early preantral mouse follicle in vitro maturation: oocyte growth, meiotic maturation and granulosa-cell proliferation. 
Theriogenology, 49:845-859.

Dean W, Santos F, Stojkovic M, Zakhartchenko V, Walter J, Wolf E, Reik W. 2001. Conservation of methylation reprogramming in mammalian development: Aberrant reprogramming in cloned embryos. Proc Natl Acad Sci, 98:13734-13738.

De Bie J, Marei WF, Maillo V, Jordaens L, Gutierrez-Adan A, Bols PE, Leroy JL. 2017. Differential effects of high and low glucose concentrations during lipolysis-like conditions on bovine in vitro oocyte quality, metabolism and subsequent embryo development. Reprod Fertil Dev. doi: 10.1071/RD16474.

Desmet KLJ, Marei WF, Merckx E, Bols PEJ, Leroy JLMR. 2016a. Effect of non-esterified fatty acids during sperm capacitation or IVF on developmental competence of bovine oocytes. Anim Reprod, 13:643.

Desmet KLJ, Van Hoeck V, Gagné D, Fournier E, Thakur A, O'Doherty AM, Walsh CP, Sirard MA, Bols PEJ, Leroy JLMR. 2016b. Exposure of bovine oocytes and embryos to elevated non-esterified fatty acid concentrations: integration of epigenetic and transcriptomic signatures in resultant blastocysts. $B M C$ Genomics, 17:1004. doi: 10.1186/s12864-016-3366-y

Folmes CDL, Dzeja PP, Nelson TJ, Terzic A. 2012. Metabolic plasticity in stem cell homeostasis and differentiation. Cell Stem Cell, 11:596-606.

Gonzalez-Recio O, Ugarte E, Bach A. 2012. Transgenerational effect of maternal lactation during pregnancy: a Holstein cow model. PLOS One, 7:1-7.

Holt WV, Fazeli A. 2010. The oviduct as a complex mediator of mammalian sperm function and selection. Mol Reprod Dev, 77:934-943.

Jordaens L, Arias-Alvarez M, Pintelon I, Thys S, Valckx S, Dezhkam Y, Bols PEJ, Leroy JLMR. 2015. Elevated non-esterified fatty acid concentrations hamper bovine oviductal epithelial cell physiology in three different in vitro culture systems. Theriogenology, 84:899-910.

Jordaens L, Van Hoeck V, Maillo V, Gutierrez-Adan A, Marei WFA, Vlaeminck B, Thys S, Sturmey RG, Bols PEJ, Leroy JLMR. 2017. Maternal metabolic stress may affect oviduct gatekeeper function. Reproduction. doi: 10.1530/REP-16-0569.

Latham KE, Schultz RM. 2001. Embryonic genome activation. Front Biosci, 6:748-759.

Lazzari G, Colleoni S, Lagutina I, Crotti G, Turini P, Tessaro I, Brunetti D, Duchi R, Galli C. 2010. Shortterm and long-term effects of embryo culture in the surrogate sheep oviduct versus in vitro culture for different domestic species. Theriogenology, 73:748-757. Leblanc S. 2010. Assessing the association of the level of milk production with reproductive performance in dairy cattle. J Reprod Dev, 56:S1-S7.

Leroy JL, Vanholder T, Delanghe JR, Opsomer G, van Soom A, Bols PE, Dewulf J, de Kruif A. 2004. Metabolic changes in follicular fluid of the dominant follicle in high-yielding dairy cows early post partum. Theriogenology 62:1131-1143.

Leroy JL, Vanholder T, Mateusen B, Christophe A, Opsomer G. 2005. Non-esterified fatty acids in follicular fluid of dairy cows and their effect on developmental capacity of bovine oocytes in vitro. Reproduction, 130:485-495.

Leroy JLMR, Van Soom A, Opsomer G, Goovaerts IG, Bols PE. 2008. Reduced fertility in high-yielding dairy cows: are the oocyte and embryo in danger? Part II. Mechanisms linking nutrition and reduced oocyte and embryo quality in high-yielding dairy cows. Reprod Domest Anim 43: 623-632.

Leroy JL, Van Hoeck V, Clemente M, Rizos D, Gutierrez-Adan A, Van Soom A, Uytterhoeven M, Bols PE. 2010. The effect of nutritionally induced hyperlipidaemia on in vitro bovine embryo quality. Hum Reprod, 25:768-778.

Lohakare JD, Südekum KH, Pattanaik AK. 2012. Nutrition-induced changes of growth from birth to first calving and its impact on mammary development and first-lactation milk yield in dairy heifers: a review. Asian-Australas J Anim Sci, 25:1338-1350.

Marei WF, Alvarez MA, Van Hoeck V, GutierrezAdan A, Bols PE, Leroy JL. 2016. Effect of nutritionally induced hyperlipidaemia on in vitro bovine embryo quality depends on the type of major fatty acid in the diet. Reprod Fertil Dev, doi: 10.1071/RD16297.

Marei WFA, De Bie J, Mohey-Elsaeed O, Wydooghe E, Bols PEJ, Leroy JLMR. 2017. Alpha-linolenic acid protects the developmental capacity of bovine cumulusoocyte complexes matured under lipotoxic conditions in vitro. Biol Reprod, doi10.1093/biolre/iox046.

Matoba S, O'Hara L, Carter F, Kelly AK, Fair T, Rizos D, Lonergan P. 2012. The association between metabolic parameters and oocyte quality early and late postpartum in Holstein dairy cows. $J$ Dairy Sci, 95:1257-1266.

Miessen K, Sharbati S, Einspanier R, Schoen J. 2011. Modelling the porcine oviduct epithelium: a polarized in vitro system suitable for long-term cultivation. Theriogenology, 76:900-910.

Sartori R, Bergfelt R, Mertens SA, Guenther JN, Parish JJ, Wiltbank MC. 2002. Fertilization and early embryonic developmentin heifers and lactating cows in summer and lactating and dry cowsin winter. J Dairy Sci, 85:2803-2812.

Sermondade N, Delarouzière V, Ravel C, Berthaut I, Verstraete L, Mathieu E, Antoine JM, Mandelbaum J. 2012. Characterization of a recurrent poor-quality embryo morphology phenotype and zygote transfer as a rescue strategy. Reprod Biomed Online, 24:403-409.

Sinclair KD, Rutherford KMD, Wallace JM, Brameld JM, Stöger R, Alberio R, Sweetman D, Gardner DS, Perry VEA, Adam CL, Ashworth CJ, Robinson JE, Dwyer CM. 2016. Epigenetics and developmental programming of welfare and production traits in farm animals. Reprod Fertil Dev, 28:1443-1478. Smith ZD, Chan MM, Mikkelsen TS, Gu H, Gnirke A, Regev A, Meissner A. 2012. A unique regulatory phase of DNA methylation in the early mammalian embryo. Nature, 484:339-344.

Smits A, De Bie J, Bols PEJ, Marei WF, Leroy JLMR. 2016. Effect of embryo culture conditions on developmental potential of bovine oocytes matured under lipotoxic conditions. In: Proceedings of the 18th International Congress on Animal Reproduction, 2016, 
Tours, France. Tours: ICAR. pp. PW552.]

Spencer TE, Johnson GA, Bazer FW, Burghardt RC. 2004. Implantation mechanisms: insights from the sheep Reproduction, 128:657-668.

Spencer TE. 2013. Early pregnancy: concepts, challenges and potential solutions. Anim Front, 3:48-55.

Sponchiado M, Gomes NS, Fontes PK, Martis T, del Collado M, Pastore A, Pugliesi G, Nogueira MFG, Binelli M. 2017. Pre-hatching embryo-dependent and independent programming of endometrial function in cattle. PLOS One. doi.org/10.1371/journal.pone.0175954.

Valckx S, Van Hoeck V, Arias-Alvarez M, Maillo V, Lopez-Cardona AP, Gutierrez-Adan A, Berth M, Cortvrindt R, Bols P, Leroy J. 2014. Elevated nonesterified fatty acid concentrations during in vitro murine follicle growth alter follicular physiology and reduce oocyte developmental competence. Fertil Steril, 102:1769-1776 e1.

Valckx SDM, Jordaens L, Cortvrindt R, Bols PEJ, Leroy JLMR. 2015. The effect of short versus long term elevated non-esterified fatty acid concentrations during murine in vitro follicle growth on oocyte developmental competence. Reprod Fertil Dev, 27:184184

Van Hoeck V, Sturmey RG, Bermejo-Alvarez P, Rizos D, Gutierrez-Adan A, Leese HJ, Bols PEJ, Leroy JLMR. 2011. Elevated non-esterified fatty acid concentrations during bovine oocyte maturation compromise early embryo physiology. PLOS One, 6:23183.
Van Hoeck V, De Bie J, Andries S, Merckx E, Bols PEJ, Leroy JLMR. 2012. Elevated concentrations of saturated NEFA during bovine in vitro embryo culture compromise pre-implantation embryo development. In: 28th Scientific Meeting European Embryo Transfer Association, 2012, Saint-Malo, France. Saint Malo: AETE.

Van Hoeck V, Leroy JLMR, Arias-Álvarez M, Rizos D, Gutierrez-Adan A, Schnorbusch K, Bols PEJ, Leese HJ, Sturmey RGS. 2013. Oocyte developmental failure in response to elevated non-esterified fatty acid concentrations: mechanistic insights. Reproduction, 145:33-44.

Van Hoeck V, Rizos D, Gutierrez-Adan A, Pintelon I, Jorssen E, Dufort I, Sirard MA, Verlaet A, Hermans N, Bols PEJ, Leroy JLMR. 2015. The interaction between differential gene expression profile and phenotype in bovine blastocysts originating from oocytes exposed to elevated non-esterified fatty acid concentrations. Reprod Fertil Dev, 27:372-384.

Van Hoeck V, Marei W, Bols PEJ, Leroy JLMR. 2016. Maternal impact of metabolic diseases: Effect of nutrient sensing pathways on developmental and differentiation programs in the bovine embryo. Anim Reprod, 13:667, 2016.

Vickers MH. 2014. Early life nutrition, epigenetics and programming of later life disease. Nutrients, 6:21652178.

Wathes DC. 2012. Mechanisms linking metabolic status and disease with reproductive outcome in the dairy cow. Reprod Domest Anim 47:304-312. 